\title{
Treatment of Chronic Aphasia: International Perspectives
}

This issue of Seminarsin Speech and Language focuses on impairment-based treatment for aphasic people who are beyond the period of "spontaneous" recovery.

A range of group and single case studies over the years demonstrates that aphasia continues to improve for many years. There is a mismatch between treatment research studies and clinical practice: for most treatment research studies, a necessary control has been to select research participants who are no longer making spontaneous recovery (i.e., without treatment); for most clinical services, impairment-based treatment ceases when the individual ceases to make significant progress. In other words, most of the evidence we have on the efficacy and effectiveness of treatment for aphasia is gathered from people who have chronic aphasia, and in the English-speaking world chronically aphasic people can expect to receive 1 hour per week of treatment on average.

When does aphasia become "chronic"? A common understanding is that the transition from acute to chronic is related to the ending of "spontaneous" recovery, and, for most, spontaneous recovery is recovery that takes place without intervention.

This definition is based on quantitative group studies of recovery, which are in good general agreement, that have determined that significant recovery can no longer be detected by $\sim 12$ months post-onset (MPO), although, of course, there are individual exceptions. This definition is the one favored, for instance, by Jacqueline Stark in her article. It differs somewhat from that of Anne Whitworth, who in her article defines chronic aphasia in terms of when spontaneous recovery is beginning to level out, drawing on Basso's (1992) review, and where the individual is needing to come to terms with a chronic condition. This is a time that often coincides with services no longer being available or provided, or when the person is considered less of a priority for active treatment. Evidence from longitudinal studies highlights that, even when treatment has ceased, recovery could still be going on, although the decision to end treatment could have been made on the basis of some evidence of lack of progress. However, recalling the mismatch between the evidence for the usefulness of treatment and the provision of services for aphasic people, previously discussed, it seems that many clinical decisions are made more on the basis of available resources than on the potential an individual has to benefit from treatment.

Whether defined in terms of quantifiable test results or the pragmatics of service provision limitations, the treatment studies described here show that people with long-term aphasia can benefit from impairment-based treatment. Each article in this special issue presents new data demonstrating that impairment-based treatment for chronic aphasia can be highly effective.

The articles are from an international spread of highly experienced aphasiologists from Austria, Canada, the United States, the United Kingdom, and Germany. Included are: a single-case treatment study (Stark) facilitating oral sentence production over a 6.6 year period; an intensive 1-month group outcome study for aphasic participants with a mean of 34 months post-onset (Code et al), which demonstrates significant gains for some, but not all participants; a computerized treatment study (Katz) including a large, multicenter

\footnotetext{
${ }^{1}$ School of Psychology, University of Exeter, Exeter, United Kingdom.

Treatment of Chronic Aphasia: International Perspectives; Guest Editor, Chris Code, Ph.D.

Semin Speech Lang 2010;31:3-4. Copyright (C) 2010
}

by Thieme Medical Publishers, Inc., 333 Seventh Avenue, New York, NY 10001, USA. Tel: +1(212) 584-4662. DOI: http://dx.doi.org/10.1055/s-0029-1244948. ISSN 0734-0478. 
Phase 3 randomized controlled trial (RCT) efficacy study showing significant gains; a computerized treatment study for chronically aphasic people (Cherney); an imaging study (Marcotte \& Ansaldo) that explores the neural changes underlying the recovery of naming abilities in three severely aphasic individuals following treatment; and the success of narrative therapy as a bridge to real-life communi- cation in two chronically aphasic individuals (Whitworth).

I am grateful to them for their contributions and to Audrey Holland for her support for this issue of Seminars in Speech and Language.

Chris Code, Ph.D. ${ }^{1}$ Guest Editor 